\title{
Enabling catalyst discovery through machine learning and high-throughput experimentation
}

Travis Williams ${ }^{1, \dagger}$, Katherine McCullough ${ }^{1, \dagger}$, and Jochen A. Lauterbach ${ }^{1,{ }^{*}}$

${ }^{1}$ Department of Chemical Engineering, University of South Carolina, Columbia, SC 29208, USA.

*Correspondence to: lauteraj@cec.sc.edu

† Authors contributed equally to this work

\section{Supplementary Materials}

\section{alculations}

The Ru-normalized rate was calculated using the following formula:

$$
\frac{\frac{3}{2} Q_{N H_{3}}\left(\frac{\rho_{N H_{3}}}{M_{N H_{3}}}\right) X_{N H_{3}}}{w_{R u} m_{c a t} M_{R u}}
$$

where $Q_{N_{3}}$ is the flow rate of ammonia, $\rho_{N_{3}}$ is the density of ammonia, $M_{N_{3}}$ is the molar mass of ammonia, $X_{N H_{3}}$ is the measured ammonia conversion, $w_{R u}$ is the weight loading of ruthenium on the catalyst, $m_{\text {cat }}$ is the mass of catalyst loaded into the reactor, and $M_{R u}$ is the molar mass of ruthenium. 


\section{Machine learning algorithm selection}

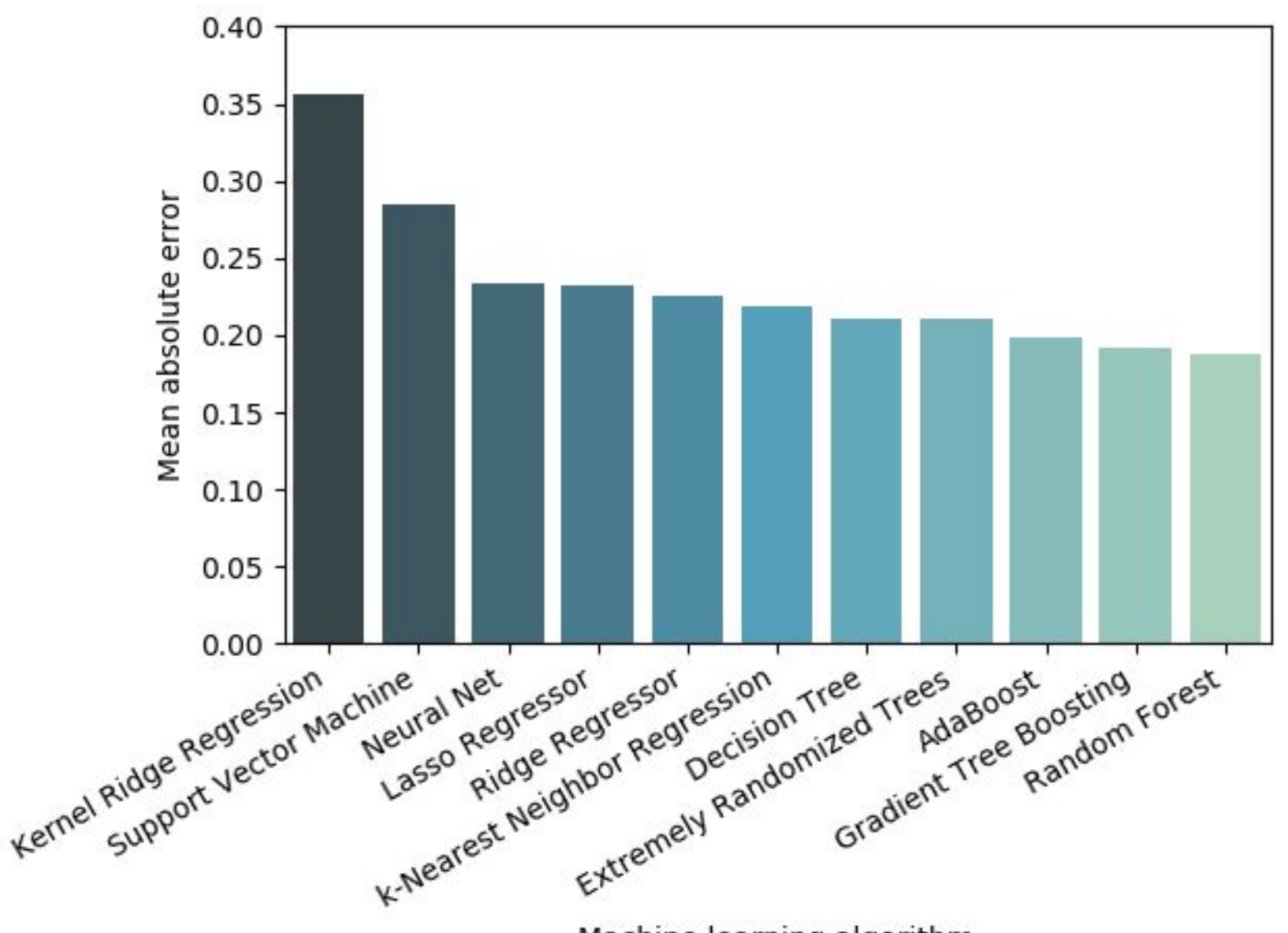

Fig. S1. Performance of machine learning algorithms. Machine learning algorithm performance was tested using the full data set (all secondary elements across all weight loadings). A full hyperparameter search was performed for each algorithm using the gridsearch algorithm from sklearn. A 3-fold crossvalidation methodology was used rather than the leave-one-out crossvalidation method to decrease the total time of the parameter search. The best combination of hyperparameters for each algorithm were used to calculate the model error. 
Table S1. Optimized hyperparameters for each algorithm on the full catalyst dataset. Values listed were optimized using a grid search method to probe all possible combinations. Unlisted parameters for each algorithm were kept at default values.

\begin{tabular}{|c|c|c|c|c|c|}
\hline Algorithm & Hyperparameter & $\begin{array}{c}\text { Optimized } \\
\text { Value }\end{array}$ & Algorithm & Hyperparameter & $\begin{array}{l}\text { Optimized } \\
\text { Value }\end{array}$ \\
\hline $\begin{array}{l}\text { Kernel Ridge } \\
\text { Regression }\end{array}$ & $\begin{array}{l}\text { Alpha } \\
\text { Coefo } \\
\text { Degree }\end{array}$ & $\begin{array}{c}1.5 \\
0 \\
2\end{array}$ & $\begin{array}{l}\text { Decision } \\
\text { Tree }\end{array}$ & $\begin{array}{c}\text { Criterion } \\
\text { Max_depth } \\
\text { Max_features } \\
\text { Min_samples_split } \\
\text { splitter }\end{array}$ & $\begin{array}{c}\text { Mse } \\
2 \\
\text { Auto } \\
5 \\
\text { Random }\end{array}$ \\
\hline $\begin{array}{l}\text { Support Vector } \\
\text { Machine }\end{array}$ & $\begin{array}{l}\text { Max_iter } \\
\text { Kernel } \\
\text { Gamma } \\
\text { Epsilon } \\
\text { Degree } \\
\text { Coefo }\end{array}$ & $\begin{array}{c}200 \\
\mathrm{Rbf} \\
1 \\
0.01 \\
2 \\
100.0\end{array}$ & $\begin{array}{c}\text { Extremely } \\
\text { Randomized } \\
\text { Trees }\end{array}$ & $\begin{array}{c}\text { N_estimators } \\
\text { Min_samples_split } \\
\text { Min_samples_leaf } \\
\text { Min_impurity_decrease } \\
\text { Max_leaf_nodes } \\
\text { Max_features } \\
\text { Max_depth } \\
\text { Criterion }\end{array}$ & $\begin{array}{c}25 \\
5 \\
2 \\
0 \\
20 \\
\text { o.1 } \\
\text { None } \\
\text { mae }\end{array}$ \\
\hline Neural Net & $\begin{array}{c}\text { Tol } \\
\text { Solver } \\
\text { Momentum } \\
\text { Max_iter } \\
\text { Learning_rate_init } \\
\text { Learning_rate } \\
\text { Hidden_layer_sizes } \\
\text { Early_stopping } \\
\text { Alpha } \\
\text { Activation }\end{array}$ & $\begin{array}{c}\text { 1e-05 } \\
\text { Lbfgs } \\
0.95 \\
500 \\
\text { o.oo1 } \\
\text { Invscaling } \\
10 \\
\text { True } \\
\text { o.oo1 } \\
\text { Tanh }\end{array}$ & Adaboost & $\begin{array}{l}\text { Base Estimator } \\
\text { Learning Rate } \\
\text { Loss } \\
\text { N_estimators }\end{array}$ & $\begin{array}{l}\text { None } \\
2 \\
\text { linear } \\
50\end{array}$ \\
\hline $\begin{array}{c}\text { Lasso } \\
\text { Regressor }\end{array}$ & $\begin{array}{c}\text { Alpha } \\
\text { Fit_intercept } \\
\text { Max_iter } \\
\text { Normalize }\end{array}$ & $\begin{array}{l}\text { 0.9 } \\
\text { False } \\
\text { 10o } \\
\text { True }\end{array}$ & $\begin{array}{l}\text { Gradient } \\
\text { Tree } \\
\text { Boosting }\end{array}$ & $\begin{array}{c}\text { Subsample } \\
\text { N_estimators } \\
\text { Min_samples_split } \\
\text { Min_samples_leaf } \\
\text { Min_impurity_decrease } \\
\text { Max_leaf_nodes } \\
\text { Max_features } \\
\text { Max_depth } \\
\text { Loss } \\
\text { Learning_rate } \\
\text { Criterion }\end{array}$ & $\begin{array}{c}1 \\
100 \\
10 \\
1 \\
0 \\
\text { None } \\
\text { Sqrt } \\
\text { None } \\
\text { Ls } \\
\text { o.1 } \\
\text { Friedman_mse }\end{array}$ \\
\hline $\begin{array}{c}\text { Ridge } \\
\text { Regressor }\end{array}$ & $\begin{array}{l}\text { Alpha } \\
\text { solver }\end{array}$ & $\begin{array}{c}1 \\
\text { sag }\end{array}$ & $\begin{array}{l}\text { Random } \\
\text { Forest }\end{array}$ & $\begin{array}{c}\text { N_estimators } \\
\text { Min_samples_split } \\
\text { Min_samples_leaf } \\
\text { Min_impurity_decrease } \\
\text { Max_leaf_nodes } \\
\text { Max_features } \\
\text { Max_depth }\end{array}$ & $\begin{array}{c}100 \\
10 \\
1 \\
0 \\
50 \\
\text { Auto } \\
5\end{array}$ \\
\hline $\begin{array}{l}\text { k-Nearest } \\
\text { Neighbor } \\
\text { Regressor }\end{array}$ & $\begin{array}{c}\text { Weights } \\
\text { P } \\
\text { N_neighbors } \\
\text { Leaf_size } \\
\text { Algorithm }\end{array}$ & $\begin{array}{c}\text { Uniform } \\
1 \\
7 \\
2 \\
\text { Ball_tree }\end{array}$ & & & \\
\hline
\end{tabular}


Table S2. Training set summary for machine learning models. Summary of training sets used in study, including catalyst compositions, target predictions, and mean absolute error.

\begin{tabular}{|c|c|c|c|c|c|}
\hline Training Set & $\begin{array}{l}\text { Catalysts in } \\
\text { Training Set }\end{array}$ & $\begin{array}{c}\text { Prediction } \\
\text { Target }\end{array}$ & $\begin{array}{c}\text { Mean Absolute } \\
\text { Error }\end{array}$ & $\begin{array}{c}\text { Root Mean } \\
\text { Squared Error }\end{array}$ & $\begin{array}{c}\text { Percent } \\
\text { Catalysts within } \\
5 \% \text { Error } \\
\end{array}$ \\
\hline $\begin{array}{l}\text { 3-catalyst } \\
\text { training set }\end{array}$ & $\begin{array}{c}\text { RuCaK, RuMnK, } \\
\text { RuInK }\end{array}$ & $\begin{array}{c}\text { Secondary } \\
\text { Elements for } \\
\text { substitution into } \\
\text { K-promoted Ru } \\
\text { catalyst }\end{array}$ & 0.157 & 0.198 & $23 \%$ \\
\hline $\begin{array}{l}\text { 22-catalyst } \\
\text { training set }\end{array}$ & $\begin{array}{c}3,1,12 \\
\text { RuCaK, RuMnK, } \\
\text { RuInK, RuCuK, } \\
\text { RuYK,RuMgK, } \\
\text { RuNiK, RuCrK, } \\
\text { RuWK, RuHfK, } \\
\text { RuScK, RuZnK, } \\
\text { RuSrK, RuBiK, } \\
\text { RuPdK, RuMoK, } \\
\text { RuRhK, RuOsK, } \\
\text { RuPtK, RuAuK, } \\
\text { RuNbK, RuFeK }\end{array}$ & $\begin{array}{c}\text { 1,3,12 and 2,2,12 } \\
\text { weight loadings } \\
\text { for secondary } \\
\text { metals substituted } \\
\text { into K-promoted } \\
\text { Ru catalyst }\end{array}$ & 0.127 & 0.169 & $20 \%$ \\
\hline \multirow[t]{3}{*}{$\begin{array}{l}\text { 28-catalyst } \\
\text { training set }\end{array}$} & $\begin{array}{c}\text { 3,1,12 } \\
\text { RuCaK, RuMnK, } \\
\text { RuInK, RuCuK, } \\
\text { RuYK,RuMgK, } \\
\text { RuNiK, RuCrK, } \\
\text { RuWK, RuHfK, } \\
\text { RuScK, RuZnK, } \\
\text { RuSrK, RuBiK, } \\
\text { RuPdK, RuMoK, } \\
\text { RuRhK, RuOsK, } \\
\text { RuPtK, RuAuK, } \\
\text { RuNbK, RuFeK }\end{array}$ & $\begin{array}{c}\text { 1,3,12 and 2,2,12 } \\
\text { weight loadings } \\
\text { for secondary } \\
\text { metals substituted } \\
\text { into K-promoted } \\
\text { Ru catalyst }\end{array}$ & 0.115 & 0.164 & $37 \%$ \\
\hline & $\begin{array}{l}\text { RuCaK, RuMnK, } \\
\text { RuInK }\end{array}$ & & & & \\
\hline & $\begin{array}{l}\text { RuCaK, RuMnK, } \\
\text { RuInK }\end{array}$ & & & & \\
\hline
\end{tabular}


Table S3 $\square$ Benchmark of ML-HTE discovered catalysts. New catalyst formulations are compared to the best catalysts reported in literature at similar conditions for $\mathrm{NH}_{3}$ decomposition.

\begin{tabular}{|c|c|c|c|c|c|c|}
\hline Catalyst & $\begin{array}{c}\mathrm{NH}_{3} \\
\text { Conversion } \\
\left(300^{\circ} \mathrm{C}\right)\end{array}$ & $\begin{array}{c}\mathrm{NH}_{3} \\
\text { Conversion } \\
\left(400^{\circ} \mathrm{C}\right)\end{array}$ & $\begin{array}{c}\mathrm{NH}_{3} \\
\%\end{array}$ & $\begin{array}{c}\text { GHSV } \\
\left(\mathrm{mL}_{\mathrm{NH}_{3}}\right. \\
\left.\mathrm{g}_{\mathrm{cat}}^{-1} \mathbf{h}^{-1}\right)\end{array}$ & $\begin{array}{c}\text { Ru-normalized } \\
\text { rate @ } 35^{\circ} \mathrm{C} \\
\left(\text { mol }_{\mathrm{H}_{2}} \mathbf{m o l}_{\left.\mathrm{Ru}^{-1} \mathbf{h}^{-1}\right)}\right.\end{array}$ & Ref. \\
\hline 3,1,12 RuYK/Al $\mathrm{O}_{2}$ & $43 \%$ & $94 \%$ & 100 & 5200 & 826.4 & a \\
\hline 3,1,12 RuMgK/Al ${ }_{2} \mathrm{O}_{3}$ & $44 \%$ & $98 \%$ & 100 & 5200 & 919.8 & $\mathrm{a}$ \\
\hline 3,1,12 RuSrK/ $/ \mathrm{Al}_{2} \mathrm{O}_{3}$ & $46 \%$ & $98 \%$ & 100 & 5200 & $879 \cdot 3$ & $\mathrm{a}$ \\
\hline 3,1,12 RuScK/ $/ \mathrm{Al}_{2} \mathrm{O}_{3}$ & $46 \%$ & $97 \%$ & 100 & 5200 & $885 \cdot 4$ & $\mathrm{a}$ \\
\hline $1,3,12 \mathrm{RuYK} / \mathrm{Al}_{2} \mathrm{O}_{3}$ & $47 \%$ & $97 \%$ & 100 & 5200 & 2702.3 & a \\
\hline 7, $20 \mathrm{RuCs} / \mathrm{CNT}$ & $28 \%$ & $92 \%$ & $\begin{array}{c}\text { Not } \\
\text { listed }\end{array}$ & 5200 & $337 \cdot 9$ & (1) \\
\hline 3,4 RuCs/CNT & $3 \%$ & $33 \%$ & $\begin{array}{c}\text { Not } \\
\text { listed }\end{array}$ & 5200 & 112.6 & (1) \\
\hline 7,7 RuNa/CNT & $10 \%$ & $100 \%$ & 30 & 6000 & 316.7 & (2) \\
\hline 7,7 RuNa/CeO ${ }_{2} \mathrm{NR}$ & $5 \%$ & $100 \%$ & 30 & 6000 & 165.6 & $(2)$ \\
\hline $7 \mathrm{Ru} / \mathrm{CeO}_{2} \mathrm{NR}$ & $8 \%$ & $71 \%$ & 30 & 6000 & 122.4 & (2) \\
\hline
\end{tabular}

a Data from this work. 
Table $S_{4}$. Catalyst features used in machine learning algorithm. Elemental properties were taken from (66). Temperature and space velocity values were measured during the experiment. Element loadings are the nominal mol fraction during synthesis. Precursor chlorine mols were calculated from the weight loading of the precursor and the number of chlorine ligands.

\begin{tabular}{cc}
\hline \multicolumn{1}{c}{ Feature Name } & \\
\hline Atomic Number & Ru Loading \\
Atomic Volume & K Loading \\
Atomic Weight & Mg Loading \\
Boiling Temperature & Ca Loading \\
Conductivity & Sc Loading \\
Covalent Radius & Ti Loading \\
Density & V Loading \\
Electron Affinity & Cr Loading \\
Electronegativity & Mn Loading \\
Fusion Enthalpy & Fe Loading \\
Ground State Energy & Co Loading \\
Heat Capacity (Mass) & Ni Loading \\
Heat Capacity (Molar) & Cu Loading \\
$1^{\text {st }}$ Ionization Energy & Zn Loading \\
$2^{\text {nd }}$ Ionization Energy & Sr Loading \\
$3^{\text {rd }}$ Ionization Energy & Y Loading \\
$4^{\text {th }}$ Ionization Energy & Zr Loading \\
$5^{\text {th }}$ Ionization Energy & Nb Loading \\
$6^{\text {th }}$ Ionization Energy & Mo Loading \\
$7^{\text {th } \text { Ionization Energy }}$ & Rh Loading \\
$8^{\text {th }}$ Ionization Energy & Pd Loading \\
Melting Temperature & Ag Loading \\
Polarizability & Cd Loading \\
Adjusted Work Function & In Loading \\
Number of Valence Electrons & Sn Loading \\
Number of s-shell Valence Electrons & Hf Loading \\
Number of p-shell Valence Electrons & Ta Loading \\
Number of d-shell Valence Electrons & W Loading \\
Number of f-shell Valence Electrons & Re Loading \\
Temperature & Os Loading \\
Space Velocity & Ir Loading \\
Precursor Chlorine Mols & Pt Loading \\
& Au Loading \\
\hline Pb Loading \\
Bi Loading \\
\hline
\end{tabular}

